Pacific

Journal of

Mathematics

COMMUTING ANALYTIC SELF-MAPS OF THE BALL

Barbara D. MacCluer

Volume $194 \quad$ No. 2

June 2000 


\title{
COMMUTING ANALYTIC SELF-MAPS OF THE BALL
}

\author{
Barbara D. MacCluer
}

\begin{abstract}
Under broad conditions, two analytic self-maps of the disk fixing 0 commute under composition precisely when they have the same Schroeder map, where the Schroeder map for an analytic $\varphi: D \rightarrow D$ with $\varphi(0)=0$ is the unique analytic function $\sigma$ on $D$ solving Schroeder's equation $\sigma \circ \varphi=\varphi^{\prime}(0) \sigma$ and satisfying $\sigma^{\prime}(0)=1$. For analytic self-maps of the ball in $C^{N}$ fixing 0 we may still seek analytic $C^{N}$-valued solutions $\sigma$ to Schroeder's equation with $\sigma^{\prime}(0)=I$, but considerable complications for existence and uniqueness of $\sigma$ may ensue. Nevertheless, we show that there are reasonably general hypotheses under which it will still be the case that two analytic self-maps of the ball fixing 0 commute if and only if they share a common Schroeder map $\sigma$ with $\sigma^{\prime}(0)=I$.
\end{abstract}

\section{Introduction.}

If $\varphi$ is an analytic map of the unit disk $D$ into itself which fixes the origin and has derivative there satisfying $0<\left|\varphi^{\prime}(0)\right|<1$ then there exists an analytic map $\sigma$ on $D$ that satisfies Schroeder's functional equation

$$
\sigma \circ \varphi=\varphi^{\prime}(0) \sigma .
$$

This "Schroeder map" $\sigma$ is unique up to constant multiples; its existence and uniqueness was proved by Koenigs in 1884 ([3]). It is usually convenient to require that $\sigma$ satisfy $\sigma^{\prime}(0)=1$. Koenigs showed that in this case $\sigma$ can be obtained as the almost uniform limit of normalized iterates of $\varphi$ :

$$
\sigma=\lim _{n \rightarrow \infty} \frac{\varphi_{n}}{\varphi^{\prime}(0)^{n}}
$$

where $\varphi_{1}=\varphi$ and $\varphi_{k+1}=\varphi \circ \varphi_{k}$. When $\varphi$ is univalent in $D, \sigma$ will be also, so that $\varphi$ is conjugate to multiplication by $\varphi^{\prime}(0)$ on $\sigma(D): \varphi=$ $\sigma^{-1} \varphi^{\prime}(0) \sigma$. Suppose $\psi$ is an analytic self-map of $D$ which commutes with $\varphi$ under composition. Then necessarily $\psi(0)=0$. Moreover, $\varphi$ and $\psi$ will have the same Schroeder maps, and conversely if $\psi: D \rightarrow D$ is analytic, fixes 0 and has the same Schroeder map as $\varphi$, then $\varphi \circ \psi=\psi \circ \varphi$. These results follow from the existence and (essential) uniqueness of the Schroeder map in one variable. $([\mathbf{1}],[\mathbf{4}]$. 
If $\varphi$ is an analytic self-map of the unit ball $B_{N}$ in $C^{N}$ which fixes the origin, then by a Schroeder map for $\varphi$ we will mean an analytic map $\sigma$ : $B_{N} \rightarrow C^{N}$ which satisfies the functional equation

$$
\sigma \circ \varphi=\varphi^{\prime}(0) \sigma
$$

where $\varphi^{\prime}(0)$ is the linear map from $C^{N}$ to $C^{N}$ given by the matrix whose $i j^{t h}$ entry is $D_{j} \varphi_{i}(0)$. By analogy to the one variable case we restrict to the case that the eigenvalues of $\varphi^{\prime}(0)$ are non-zero and of modulus strictly less than 1. In addition we exclude maps which are "unitary on a slice" of the ball; that is, maps $\varphi$ for which there exists $\zeta, \eta$ in $\partial B_{N}$ so that $\varphi(\lambda \zeta)=\lambda \eta$ for all $\lambda \in D$. We are chiefly interested in Schroeder maps $\sigma$ which are locally univalent near 0 . This is equivalent to requiring that $\sigma^{\prime}(0)$ be invertible ( $[\mathbf{5}$, 1.3.7 and 15.1.8]). In fact when there is a solution to Equation (1) with $\sigma^{\prime}(0)$ invertible, there will be a solution with $\sigma^{\prime}(0)=I$. Precise conditions under which such a solution exists for a given $\varphi$ are known ([2]; see also Theorem 1 and Corollary 2 below), but are somewhat complicated. A basic issue is whether any algebraic relationships of the form

$$
\lambda_{j}=\lambda_{1}^{k_{1}} \lambda_{2}^{k_{2}} \cdots \lambda_{N}^{k_{N}}
$$

hold between the eigenvalues $\lambda_{k}$ of $\varphi^{\prime}(0)$, where $k_{i} \geq 0$ and $\sum k_{i} \geq 2$, and if any such relationships do hold, whether they in fact prevent the existence of a locally univalent Schroeder map. Such an algebraic relationship for an eigenvalue of $\varphi$ will be called a resonance of $\varphi$.

The results which make this precise are as follows. As a convenient normalization we may assume, by a unitary change of variables, that $\varphi^{\prime}(0)$ is upper triangular.

Theorem 1 ([2]). Suppose $\varphi$ is an analytic map of $B_{N}$ into $B_{N}$ with $\varphi(0)=$ 0 and $A=\varphi^{\prime}(0)$ an upper triangular diagonalizable matrix, with diagonal entries $\lambda_{1}, \lambda_{2}, \ldots, \lambda_{N}$ such that $0<\left|\lambda_{j}\right|<1$. Assume further that $\varphi$ is not unitary on any slice. Suppose that $\lambda_{j}=\lambda_{1}^{k_{1}} \cdots \lambda_{N}^{k_{N}}$ is the longest expression (maximal $\left.\sum k_{i}\right)$ for one eigenvalue of $A$ as a product of any number of the eigenvalues of $A$. Set $m=k_{1}+\cdots+k_{N}$ and $M=$ the number of multi-indices for $C^{N}$ of total order less than or equal to $m$. Let $\mathcal{M}$ be the upper left $M \times M$ corner of the matrix for the composition operator $C_{\varphi}$ with respect to the standard (non-normalized) basis for any weighted Hardy space $H_{\beta}^{2}\left(B_{N}\right)$, ordered in the usual way. If $\mathcal{M}$ is diagonalizable, then Schroeder's Equation (1) has a solution $\sigma$ with $\sigma^{\prime}(0)=I$.

The "standard basis" referred to in this theorem consists of the monomials $1, z_{1}, z_{2}, \ldots, z_{n}, z_{1}^{2}, z_{1} z_{2}, \ldots$ ordered as follows: $z^{\alpha}$ precedes $z^{\gamma}$ where $\alpha=$ $\left(\alpha_{1}, \ldots, \alpha_{N}\right)$ and $\gamma=\left(\gamma_{1}, \ldots, \gamma_{N}\right)$ are multi-indices, if either $|\alpha|<|\gamma|$ or, in the case $|\alpha|=|\gamma|$, if there is a $j_{0}$ so that $\alpha_{j}=\gamma_{j}$ for $j<j_{0}$ and $\alpha_{j_{0}}>\gamma_{j_{0}}$. The matrix for the composition operator $C_{\varphi}$ with respect to this basis has as 
its $j^{\text {th }}$ column the coefficients of $\varphi^{\alpha}$ with respect to this basis, where $z^{\alpha}$ is the $j^{\text {th }}$ monomial in the prescribed ordering. A weighted Hardy space $H_{\beta}^{2}\left(B_{N}\right)$ is a Hilbert space of analytic functions on $B_{N}$ for which the monomials form a complete orthogonal set of non-zero vectors satisfying

$$
\beta\left(\left|\alpha_{1}\right|\right) \equiv \frac{\left\|z^{\alpha_{1}}\right\|}{\left\|z^{\alpha_{1}}\right\|_{2}}=\frac{\left\|z^{\alpha_{2}}\right\|}{\left\|z^{\alpha_{2}}\right\|_{2}}
$$

whenever $\left|\alpha_{1}\right|=\left|\alpha_{2}\right|$, where $\|\cdot\|$ denotes the norm in $H_{\beta}^{2}\left(B_{N}\right)$ and $\|\cdot\|_{2}$ denotes the norm in $L^{2}\left(\sigma_{N}\right), \sigma_{N}$ being normalized Lebesgue measure on $B_{N}$. When $\varphi(0)=0$ and $\varphi$ is not unitary on any slice there exist weighted Hardy spaces on which the composition operator $C_{\varphi}$ (defined by $C_{\varphi}(f)=f \circ \varphi$ ) is a compact operator $([\mathbf{2}])$.

There is a converse to Theorem 1 which says, under the same hypotheses on $\varphi$, that if $\varphi$ has a Schroeder map with invertible derivative at the origin, then every upper left corner of the matrix for $C_{\varphi}$ is diagonalizable.

While we won't have direct need for the full strength of Theorem 1 here, the following corollary will play a crucial role in our study of commuting analytic self-maps of $B_{N}$. It gives a description of all Schroeder maps (locally univalent or not) for $\varphi$, based on the presence or absence of resonances for $\varphi$.

Corollary $2([\mathbf{2}])$. Suppose the hypotheses of Theorem 1 hold and that in addition $A=\varphi^{\prime}(0)$ is diagonal. Then all solutions to Schroeder's Equation (1) can be described as $f=g \circ \sigma$ where $\sigma$ is a Schroeder map with $\sigma^{\prime}(0)=$ $I$, as given in Theorem 1 and $g=\left(g_{1}, g_{2}, \ldots, g_{N}\right)$ is a mapping on $C^{N}$ with polynomial coordinate functions. Moreover, if $g_{k}=\sum c(\gamma) z^{\gamma}$, then the coefficients $c(\gamma), \gamma=\left(\gamma_{1}, \ldots, \gamma_{N}\right)$ are 0 unless $\lambda_{k}=\lambda_{1}^{\gamma_{1}} \lambda_{2}^{\gamma_{2}} \cdots \lambda_{N}^{\gamma_{N}}\left(\gamma_{i} \geq 0\right)$, in which case $c(\gamma)$ can be chosen arbitrarily.

If $A=\varphi^{\prime}(0)$ is merely diagonalizable, with $S A S^{-1}=\operatorname{diag}\left(\lambda_{1}, \lambda_{2}, \ldots, \lambda_{N}\right)$, then an arbitrary Schroeder map has the form $S^{-1} \circ g \circ S \circ \sigma$ with $\sigma$ and $g$ as just described.

Note that $g_{k}$ always includes a linear term $b_{k} z_{k}$ ( $b_{k}$ arbitrary), and if $\lambda_{k}$ is a repeated eigenvalue of $\varphi^{\prime}(0)$ there will be other linear terms with arbitrary coefficients. The terms of $g_{k}$ with order at least two correspond to the resonance relations for $\lambda_{k}$. When no resonance relations hold, $g$ is linear. We emphasize that a resonance relation expresses an eigenvalue $\lambda_{j}$ as a product $\lambda_{1}^{k_{1}} \lambda_{2}^{k_{2}} \cdots \lambda_{N}^{k_{N}}$ where $\sum k_{i} \geq 2$; a relation $\lambda_{j}=\lambda_{k}$ is not a resonance relation.

The goal of this paper is to study commuting analytic self-maps of $B_{N}$ and to see, by analogy with known results in one variable, to what extent it still is the case that commuting maps are those which share a locally univalent Schroeder map. Our main results (Theorems 3 and 7) will show that under natural hypotheses on $\varphi$, a map $\psi$ which commutes with $\varphi$ and 
has no resonances in common with $\varphi$ will share a locally univalent Schroeder map with $\varphi$. Examples will be give to show that this can fail if $\varphi$ and $\psi$ have resonances in common.

\section{Non-resonant maps.}

In studying the Schroeder maps for commuting self-maps $\varphi, \psi$ of $B_{N}$, the easiest situation arises when at least one of $\varphi, \psi$ has no resonances. This means, say, that no eigenvalue of $\varphi^{\prime}(0)$ can be written as a product of two or more of the other eigenvalues, although repeated eigenvalues are allowed.

Theorem 3. Suppose $\varphi: B_{N} \rightarrow B_{N}$ is analytic, with $\varphi(0)=0$. Assume that $A=\varphi^{\prime}(0)$ is upper triangular diagonalizable with eigenvalues $\lambda_{1}, \lambda_{2}, \ldots, \lambda_{N}, 0<\left|\lambda_{j}\right|<1$. Assume further that $\varphi$ is not unitary on any slice and that no resonance relations hold for any of the $\lambda_{j}$ 's. If $\psi: B_{N} \rightarrow$ $B_{N}$ is analytic and $\psi \circ \varphi=\varphi \circ \psi$ then $\varphi$ and $\psi$ share a common Schroeder map which is locally univalent near 0.

Proof. Since $\varphi$ is not unitary on any slice and $\varphi \psi(0)=\psi \varphi(0)=\psi(0)$ we must have $\psi(0)=0$, since the fixed point set of $\varphi$ in $B_{N}$ is affine $([5,8.2 .3])$. By the $m=1, M=N+1$ case of Theorem 1 we know that $\varphi$ has a Schroeder $\operatorname{map} \sigma_{\varphi}$ with $\sigma_{\varphi}^{\prime}(0)=I$. Moreover,

$$
\left(\sigma_{\varphi} \circ \psi\right) \circ \varphi=\sigma_{\varphi} \circ \varphi \circ \psi=\varphi^{\prime}(0)\left(\sigma_{\varphi} \circ \psi\right)
$$

so $\sigma_{\varphi} \circ \psi$ is a Schroeder map for $\varphi$. By Corollary 2 this tells us that $\sigma_{\varphi} \circ \psi=$ $S^{-1} B S \sigma_{\varphi}$ where $S$ diagonalizes $\varphi^{\prime}(0)$ and $B$ is linear. Differentiation of this equation gives $\sigma_{\varphi}^{\prime}(0) \psi^{\prime}(0)=S^{-1} B S \sigma_{\varphi}^{\prime}(0)$ so that in fact $S^{-1} B S=\psi^{\prime}(0)$ and $\sigma_{\varphi}$ is a Schroeder map for both $\varphi$ and $\psi$, with derivative at 0 equal to $I$.

It need not be the case that $\varphi$ and $\psi$ have the same set of Schroeder maps; see Example 1 in the next section.

As a converse to this result we have the following theorem.

Theorem 4. Suppose $\varphi, \psi$ are analytic self-maps of $B_{N}$, each fixing 0 , with $\varphi^{\prime}(0) \psi^{\prime}(0)=\psi^{\prime}(0) \varphi^{\prime}(0)$. Suppose further that there exists an analytic $\sigma$ : $B_{N} \rightarrow C^{N}$ with $\sigma^{\prime}(0)$ invertible and both $\sigma \circ \varphi=\varphi^{\prime}(0) \sigma$ and $\sigma \circ \psi=\psi^{\prime}(0) \sigma$. Then $\varphi \circ \psi=\psi \circ \varphi$.

Proof. Since $\sigma$ is locally univalent near 0 we may write

$$
\varphi=\sigma^{-1} \varphi^{\prime}(0) \sigma
$$

and

$$
\psi=\sigma^{-1} \psi^{\prime}(0) \sigma
$$

in an open neighborhood of 0 . Thus near 0 we have $\varphi \circ \psi=\sigma^{-1} \varphi^{\prime}(0) \sigma \sigma^{-1} \psi^{\prime}(0) \sigma=\sigma^{-1} \varphi^{\prime}(0) \psi^{\prime}(0) \sigma=\sigma^{-1} \psi^{\prime}(0) \varphi^{\prime}(0) \sigma=\psi \circ \varphi$. 
Since $\varphi \circ \psi=\psi \circ \varphi$ in an open neighborhood of 0 and the compositions are defined on $B_{N}$ we must have $\varphi \circ \psi=\psi \circ \varphi$ in $B_{N}$.

The last result need not hold if the hypothesis on the commutability of the derivatives at 0 is omitted: Take $\varphi, \psi$ to be linear maps which do not commute. They share $\sigma(z)=z$ as a common locally univalent Schroeder map.

\section{Resonances.}

We begin with several examples which will help set the stage for Theorem 7 , the main result of this section.

Example 1. Let $\varphi\left(z_{1}, z_{2}\right)=\left(c_{1} z_{1}, c_{1}^{3} z_{2}+c_{2} z_{1}^{2}\right)$ where $c_{1}, c_{2}$ are sufficiently small non-zero constants so that $\varphi\left(B_{2}\right) \subset B_{2}$. Note that

$$
\varphi^{\prime}(0)=\left(\begin{array}{cc}
c_{1} & 0 \\
0 & c_{1}^{3}
\end{array}\right)
$$

and the resonance $\lambda_{2}=\lambda_{1}^{3}$ holds for the eigenvalues $\lambda_{1}=c_{1}, \lambda_{2}=c_{1}^{3}$ of $\varphi^{\prime}(0)$. It is easy to check that

$$
\sigma_{\varphi}=\left(z_{1}, z_{2}+\frac{c_{2}}{c_{1}^{3}-c_{1}^{2}} z_{1}^{2}\right)
$$

is a Schroeder map for $\varphi$ with derivative at 0 equal to $I$ (this example is also discussed in [2]). By Corollary 2 all Schroeder maps for $\varphi$ are of the form $g \circ \sigma_{\varphi}$ where $g$ is a polynomial map $\left(b_{1} z_{1}, b_{2} z_{2}+b_{3} z_{1}^{3}\right)$ for arbitrary constants $b_{1}, b_{2}$ and $b_{3}$, and thus have the form

$$
\left(b_{1} z_{1}, b_{2} z_{2}+\frac{b_{2} c_{2}}{c_{1}^{3}-c_{1}^{2}} z_{1}^{2}+b_{3} z_{1}^{3}\right) .
$$

Now suppose that $\psi$ commutes with $\varphi$. We know from the calculations in Theorem 3 that $\sigma_{\varphi} \circ \psi$ is a Schroeder map for $\varphi$ and hence $\sigma_{\varphi} \circ \psi=g \circ \sigma_{\varphi}$ for $g$ as above. From this we easily determine that $\psi$ must be of the form

$$
\left(b_{1} z_{1}, b_{2} z_{2}+\frac{c_{2}}{c_{1}^{3}-c_{1}^{2}}\left(b_{2}-b_{1}^{2}\right) z_{1}^{2}+b_{3} z_{1}^{3}\right)
$$

for some constants $b_{1}, b_{2}, b_{3}$, and moreover any choice of these constants will give a map which commutes with $\varphi$. If these constants are chosen sufficiently small, $\psi\left(B_{2}\right) \subset B_{2}$. Note that whenever $b_{3} \neq 0$ we have a commuting map which is not an iterate of $\varphi$, so the set of maps which commute with $\varphi$ is considerably larger than just the natural iterates of $\varphi$.

If $b_{2} \neq b_{1}^{3}$ then

$$
\left(z_{1}, z_{2}+\frac{c_{2}}{c_{1}^{3}-c_{1}^{2}} z_{1}^{2}+\frac{b_{3}}{b_{2}-b_{1}^{3}} z_{1}^{3}\right)
$$


is a common Schroeder map for $\varphi$ and $\psi$ with derivative at 0 equal to $I$. We remark that

$$
\left(z_{1}, z_{2}+\frac{c_{2}}{c_{1}^{3}-c_{1}^{2}} z_{1}^{2}+\alpha z_{1}^{3}\right)
$$

where $\alpha \neq b_{3} /\left(b_{2}-b_{1}^{3}\right)$ is a Schroeder map for $\varphi$ but not for $\psi$, so that while $\varphi$ and $\psi$ have a locally univalent Schroeder map in common, their sets of Schroeder maps are not the same.

On the other hand, if $b_{2}=b_{1}^{3}, b_{3} \neq 0$ and

$$
\psi\left(z_{1}, z_{2}\right)=\left(b_{1} z_{1}, b_{2} z_{2}+\frac{c_{2}}{c_{1}^{3}-c_{1}^{2}}\left(b_{2}-b_{1}^{2}\right) z_{1}^{2}+b_{3} z_{1}^{3}\right)
$$

then $\psi$ commutes with $\varphi$ but $\psi$ has no locally univalent Schroeder map by the converse of Theorem 1 . One can check that the upper left $7 \times 7$ corner of the matrix for $C_{\psi}$ has diagonal entries $1, b_{1}, b_{1}^{3}, b_{1}^{2}, b_{1}^{4}, b_{1}^{6}, b_{1}^{3}$ and three non-zero off diagonal entries: $c_{2}\left(b_{1}^{3}-b_{1}^{2}\right) /\left(c_{1}^{3}-c_{1}^{2}\right)$ in the $4-3$ position, $c_{2}\left(b_{1}^{3}-b_{1}^{2}\right) b_{1} /\left(c_{1}^{3}-c_{1}^{2}\right)$ in the $7-5$ position, and $b_{3} \neq 0$ in the 7-3 position. This matrix is not diagonalizable. Note that the situation being considered here is that of the resonances of $\varphi$ also being resonances of $\psi$, where $\psi$ is not a natural iterate of $\varphi$.

We also note that this example shows that two self-maps of the ball which each commute with $\varphi$ need not commute with each other as

$$
\psi_{1}\left(z_{1}, z_{2}\right)=\left(b_{1} z_{1}, b_{2} z_{2}+\frac{c_{2}}{c_{1}^{3}-c_{1}^{2}}\left(b_{2}-b_{1}^{2}\right) z_{1}^{2}+b_{3} z_{1}^{3}\right)
$$

and

$$
\psi_{2}\left(z_{1}, z_{2}\right)=\left(b_{1} z_{1}, b_{2} z_{2}+\frac{c_{2}}{c_{1}^{3}-c_{1}^{2}}\left(b_{2}-b_{1}^{2}\right) z_{1}^{2}+\frac{1}{2} b_{3} z_{1}^{3}\right)
$$

both commute with $\varphi$ but fail to commute with each other if $b_{1}, b_{2}$ and $b_{3}$ are chosen to be sufficiently small non-zero values with $b_{1}^{3} \neq b_{2}$.

In two variables only one resonance relation is possible (either $\lambda_{1}=\lambda_{2}^{n}$ or $\left.\lambda_{2}=\lambda_{1}^{m}\right)$, but as the number of dimensions increases so do the possible variety of resonance equations. The next example, describing a general situation in $C^{3}$ will be instructive for formulating a general theorem.

Example 2. Consider any analytic mapping $\varphi: B_{3} \rightarrow B_{3}$, fixing 0 and not unitary on any slice, where $\varphi^{\prime}(0)$ is diagonal, with diagonal entries $\lambda_{j}$ satisfying $1>\left|\lambda_{1}\right|>\left|\lambda_{2}\right|>\left|\lambda_{3}\right|>0$ where the resonances

$$
\begin{aligned}
\lambda_{2}=\lambda_{1}^{n},(n \geq 2) \quad \text { and } \\
\lambda_{3}=\lambda_{1}^{m} \lambda_{2}^{k}=\lambda_{1}^{m+n k}(m, k \geq 0, m+k \geq 2, \text { and } m<n)
\end{aligned}
$$


hold. Notice that we, in fact, have $k+1$ different resonances for $\lambda_{3}$ :

$$
\lambda_{3}=\lambda_{1}^{m+n k}=\lambda_{1}^{r_{1}} \lambda_{2}=\lambda_{1}^{r_{2}} \lambda_{2}^{2}=\cdots=\lambda_{1}^{r_{k}} \lambda_{2}^{k}
$$

where

$$
r_{j}+j n=m+n k
$$

for $j=1, \ldots, k$. If $\varphi$ satisfies the hypotheses of Corollary 2 then all Schroeder maps are of the form $g \circ \sigma_{\varphi}$ where $\sigma_{\varphi}$ is a Schroeder map satisfying $\sigma_{\varphi}^{\prime}(0)=I$ and $g$ is a polynomial mapping with

$$
g_{1}=b_{1} z_{1}, g_{2}=b_{2} z_{2}+c_{1} z_{1}^{n}
$$

and

$$
g_{3}=b_{3} z_{3}+c_{2} z_{1}^{m+n k}+c_{3} z_{1}^{r_{1}} z_{2}+c_{4} z_{1}^{r_{2}} z_{2}^{2}+\cdots+c_{k+2} z_{1}^{r_{k}} z_{2}^{k}
$$

for arbitrary choice of the coefficients. Denote the collection of all such polynomial maps $\mathcal{G}_{\varphi}$.

Now suppose $\psi: B_{3} \rightarrow B_{3}$ commutes with $\varphi$ and that no resonance of $\varphi$ is also a resonance of $\psi$. We know $\sigma_{\varphi} \circ \psi$ is a Schroeder map for $\varphi$ so $\sigma_{\varphi} \circ \psi=g \circ \sigma_{\varphi}$ for some $g \in \mathcal{G}_{\varphi}$. Taking derivatives, we see that $\sigma_{\varphi}^{\prime}(0) \psi^{\prime}(0)=$ $g^{\prime}(0) \sigma_{\varphi}^{\prime}(0)$ and thus $\psi^{\prime}(0)=g^{\prime}(0)=\operatorname{diag}\left(b_{1}, b_{2}, b_{3}\right)$. Our hypothesis on the resonances of $\psi$ implies that $b_{2} \neq b_{1}^{n}, b_{3} \neq b_{1}^{m+n k}, b_{3} \neq b_{1}^{r_{1}} b_{2}, \ldots, b_{3} \neq b_{1}^{r_{k}} b_{2}^{k}$.

We claim that there exists $\hat{g}$ in $\mathcal{G}_{\varphi}$ with $\hat{g}^{\prime}(0)=I$ solving $\hat{g} \circ g=g^{\prime}(0) \hat{g}$. Once the claim is verified we see the following holds in a neighborhood of 0 :

$$
\begin{aligned}
\left(\hat{g} \circ \sigma_{\varphi}\right) \circ \psi & =\left(\hat{g} \circ \sigma_{\varphi}\right) \circ \sigma_{\varphi}^{-1} \circ g \circ \sigma_{\varphi}=\hat{g} \circ g \circ \sigma_{\varphi} \\
& =g^{\prime}(0) \circ \hat{g} \circ \sigma_{\varphi}=\psi^{\prime}(0)\left(\hat{g} \circ \sigma_{\varphi}\right)
\end{aligned}
$$

since $\psi=\sigma_{\varphi}^{-1} g \sigma_{\varphi}$ near 0 . If $\left(\hat{g} \circ \sigma_{\varphi}\right) \circ \psi=\phi^{\prime}(0)\left(\hat{g} \circ \sigma_{\varphi}\right)$ holds near 0 , then it holds in $B_{3}$ since $\hat{g}$ is defined on $C^{3}$. This shows that $\hat{g} \circ \sigma_{\varphi}$ is a Schroeder map for $\psi$ with derivative at 0 equal to $I$; it is also a Schroeder map for $\varphi$ by Corollary 2 .

To verify the claim we will show that coefficients $\hat{c}_{1}, \hat{c}_{2}, \ldots, \hat{c}_{k+2}$ may be determined so that $\hat{g}$ given by

$$
\hat{g}_{1}=z_{1}, \hat{g}_{2}=z_{2}+\hat{c}_{1} z_{1}^{n}
$$

and

$$
\hat{g}_{3}=z_{3}+\hat{c}_{2} z_{1}^{m+n k}+\hat{c}_{3} z_{1}^{r_{1}} z_{2}+\hat{c}_{4} z_{1}^{r_{2}} z_{2}^{2}+\cdots+\hat{c}_{k+2} z_{1}^{r_{k}} z_{2}^{k}
$$

solves $\hat{g} \circ g=g^{\prime}(0) \hat{g}$. Notice that $\hat{g}_{1} \circ g=g_{1}=b_{1} z_{1}=b_{1} \hat{g}_{1}$ and that $\hat{g}_{2} \circ g=b_{2} \hat{g}_{2}$ provided $\hat{c}_{1}=c_{1} /\left(b_{2}-b_{1}^{n}\right)$; the hypothesis $b_{2} \neq b_{1}^{n}$ being used here.

Finally, we turn to 
Using the forms of $\hat{g}_{3}$ and $g$, we expand the left side of Equation (3) into a sum of monomials and observe that each of these monomials is a scalar multiple of a monomial which also appears in $b_{3} \hat{g}_{3}$, the right side of Equation (3). To see this, observe that when we expand $g_{1}^{r_{j}} g_{2}^{j}=\left(b_{1} z_{1}\right)^{r_{j}}\left(b_{2} z_{2}+c_{1} z_{1}^{n}\right)^{j}$ we get terms which are scalar multiples of the monomials $z_{1}^{r_{j}} z_{2}^{s}\left(z_{1}^{n}\right)^{j-s}=$ $z_{1}^{r_{j}+n(j-s)} z_{2}^{s}(0 \leq s \leq j)$. Since $r_{j}+n(j-s)=r_{s}$, this monomial, with some scalar coefficient, appears in $b_{3} \hat{g}_{3}$.

By equating in turn the coefficients of

$$
z_{1}^{r_{k}} z_{2}^{k}, z_{1}^{r_{k-1}} z_{2}^{k-1}, \ldots, z_{1}^{r_{1}} z_{2}, z_{1}^{m+n k},
$$

we obtain equations for the unknown coefficients $\hat{c}_{k+2}, \hat{c}_{k+1}, \ldots, \hat{c}_{2}$. The equation obtained from the coefficients of $z_{1}^{r_{k}} z_{2}^{k}$ is

$$
c_{k+2}+\hat{c}_{k+2} b_{2}^{k} b_{1}^{r_{k}}=b_{3} \hat{c}_{k+2}
$$

which may be solved for $\hat{c}_{k+2}$ provided $b_{3} \neq b_{1}^{r_{k}} b_{2}^{k}$; this is guaranteed by the hypothesis on the resonances of $\psi$. Continuing, suppose that by comparing the coefficients of $z_{1}^{r_{k}} z_{2}^{k}, z_{1}^{r_{k-1}} z_{2}^{k-1}, \ldots, z_{1}^{r_{j+1}} z_{2}^{j+1}$ the coefficients $\hat{c}_{k+2}, \hat{c}_{k+1}, \ldots, \hat{c}_{j+3}$ have been determined. Next we compare coefficients of $z_{1}^{r_{j}} z_{2}^{j}$ on both sides of Equation (3). None of the terms

$$
\hat{c}_{2} g_{1}^{m+n k}, \hat{c}_{3} g_{1}^{r_{1}} g_{2}, \ldots, \hat{c}_{j+1} g_{1}^{r_{j-1}} g_{2}^{j-1}
$$

contribute any terms of the form $z_{1}^{r_{j}} z_{2}^{j}$. The expansion of $\hat{c}_{j+2} g_{1}^{r_{j}} g_{2}^{j}$ contributes a term $\hat{c}_{j+2} b_{1}^{r_{j}} b_{2}^{j} z_{1}^{r_{j}} z_{2}^{j}$. The expansions of

$$
\hat{c}_{j+3} g_{1}^{r_{j+1}} g_{2}^{j+1}, \ldots, \hat{c}_{k+2} g_{1}^{r_{k}} g_{2}^{k}
$$

contribute terms $z_{2}^{r_{j}} z_{2}^{j}$ all of whose coefficients involve the previously determined coefficients $\hat{c}_{j+3}, \ldots, \hat{c}_{k+2}$ (and $b_{1}, b_{2}$ ). Thus equating the coefficients of $z_{1}^{r_{j}} z_{2}^{j}$ on both sides of Equation (3) leads to an equation of the form

$$
c_{j+2}+\hat{c}_{j+2} b_{1}^{r_{j}} b_{2}^{j}+\text { known terms }=b_{3} \hat{c}_{j+2}
$$

where "known terms" refers to a sum involving the known values $\hat{c}_{j+3}, \ldots$, $\hat{c}_{k+2}$ and the $b_{i}{ }^{\prime} s$. This equation may be solved for $\hat{c}_{j+2}$ provided $b_{3} \neq b_{1}^{r_{j}} b_{2}^{j}$, which is part of our hypothesis. Continuing this process we determine all of the coefficients of the second and higher order terms of $\hat{g}_{j}$. Note that the only first order term in $\hat{g}_{3} \circ g$ is $b_{3} z_{3}$ and this is the only first order term on the right side of Equation (3). Thus we have found a choice of coefficients so that $\hat{g} \circ g=g^{\prime}(0) \hat{g}$, verifying the claim.

We set some notation and terminology which will be useful in the main result. We now restrict attention to the case that the eigenvalues $\varphi^{\prime}(0)$ are distinct, non-zero, and of modulus less than 1 . There is no loss of generality in assuming that $\varphi^{\prime}(0)$ is upper triangular, with diagonal entries $\lambda_{1}, \lambda_{2}, \ldots, \lambda_{N}$ satisfying $1>\left|\lambda_{1}\right| \geq\left|\lambda_{2}\right| \geq \cdots \geq\left|\lambda_{N}\right|>0$, since there is a 
unitary map $U$ so that $U^{*} \varphi^{\prime}(0) U$ is upper triangular with the eigenvalues of $\varphi^{\prime}(0)$ appearing in the prescribed order. Moreover, if $\varphi$ and $\psi$ commute, then so do $U^{*} \varphi U$ and $U^{*} \psi U$, and $\varphi$ and $\psi$ have a common locally univalent Schroeder map if and only if $U^{*} \varphi U$ and $U^{*} \psi U$ do. This ordering on the eigenvalues of $\varphi^{\prime}(0)$ implies that $\lambda_{1}$ has no resonance relations, and in general a resonance for $\lambda_{j}$ is of the form

$$
\lambda_{j}=\lambda_{1}^{k_{1}} \lambda_{2}^{k_{2}} \cdots \lambda_{j-1}^{k_{j-1}}
$$

where $k_{i} \geq 0$ and $\sum k_{i} \geq 2$.

For $j \geq 2$ we say that a monomial $c z_{1}^{k_{1}} z_{2}^{k_{2}} \cdots z_{j-1}^{k_{j-1}}$ ( $c$ any non-zero scalar) is $j$-permissible (for $\varphi$ ) if

$$
\lambda_{j}=\lambda_{1}^{k_{1}} \lambda_{2}^{k_{2}} \cdots \lambda_{j-1}^{k_{j-1}}
$$

call the corresponding multi-index $\left(k_{1}, k_{2}, \ldots, k_{j-1}, 0, \ldots, 0\right) \mathrm{j}$-permissible as well. There is a one-to-one correspondence between a resonance for $\lambda_{j}$ and a $\mathrm{j}$-permissible monomial with scalar coefficient 1 (or a j-permissible multi-index). For a given $\varphi$, let $\Gamma_{j}$ denote the j-permissible multi-indices, so that $\left(k_{1}, k_{2}, \ldots, k_{j-1}, 0, \ldots, 0\right) \in \Gamma_{j}$ if and only if $\lambda_{j}=\lambda_{1}^{k_{1}} \lambda_{2}^{k_{2}} \cdots \lambda_{j-1}^{k_{j-1}}$ and $\Gamma_{j}$ is empty if $\lambda_{j}$ has no resonance relations. We order the multi-indices in $\Gamma_{j}$ according to the following rule: A multi-index $\alpha$ in $\Gamma_{j}$ preceedes a multi-index $\beta$ if either the $k_{j-1}$ entry of $\alpha$ is greater than the $k_{j-1}$ entry of $\beta$, or if the entries in the $k_{i}$ through $k_{j-1}$ positions agree for some $i<j$, then the $k_{i-1}$ entry of $\alpha$ is greater than the $k_{i-1}$ entry of $\beta$. This is not the "usual" ordering on multi-indices. For example, if $\varphi$ has resonance relations $\lambda_{2}=\lambda_{1}^{2}, \lambda_{3}=\lambda_{1}^{3}=\lambda_{1} \lambda_{2}$ and

$$
\lambda_{4}=\lambda_{3}^{2} \lambda_{1}=\lambda_{2}^{2} \lambda_{3}=\lambda_{1}^{2} \lambda_{2} \lambda_{3}=\lambda_{2}^{3} \lambda_{1}=\lambda_{1}^{4} \lambda_{3}=\lambda_{2}^{2} \lambda_{1}^{3}=\lambda_{1}^{5} \lambda_{2}=\lambda_{1}^{7}
$$

then the ordering on $\Gamma_{4}$ is

$$
\begin{aligned}
& (1,0,2,0),(0,2,1,0),(2,1,1,0),(4,0,1,0) \\
& (1,3,0,0),(3,2,0,0),(5,1,0,0),(7,0,0,0) .
\end{aligned}
$$

Recall the notation $\mathcal{G}_{\varphi}$ is used for the collection of all polynomial mappings $g=\left(g_{1}, g_{2}, \ldots, g_{N}\right)$ where

$$
g_{j}\left(z_{1}, \ldots, z_{N}\right)=b_{j} z_{j}+\sum_{\gamma \in \Gamma_{j}} c^{j}(\gamma) z^{\gamma}
$$

where the coefficients $b_{j}$ and $c^{j}(\gamma)$ are arbitrary.

Lemma 5. With $\varphi$ as just described, suppose $g \in \mathcal{G}_{\varphi}$ and $\hat{g} \in \mathcal{G}_{\varphi}$ with $\hat{g}^{\prime}(0)=I$. Then the monomials of order at least two in the expansion of $\hat{g}_{j} \circ g$ are all $j$-permissible, for $j \geq 2$. 
Proof. The coordinate functions $\hat{g}_{j}$ are of the form

$$
\hat{g}_{j}=z_{j}+\sum \hat{c}^{j}(\gamma) z^{\gamma}
$$

where the sum is over all multi-indices $\gamma$ in $\Gamma_{j}$. Thus

$$
\hat{g}_{j} \circ g=g_{j}+\sum_{\Gamma_{j}} \hat{c}^{j}(\gamma) g^{\gamma}
$$

and it suffices to show that each monomial in the expansion of $g^{\gamma}$ is jpermissible. Consider $g^{\gamma}$ where $\gamma=\left(\gamma_{1}, \gamma_{2}, \ldots, \gamma_{j-1}, 0, \ldots, 0\right), \gamma_{i} \geq 0$, $\sum \gamma_{i} \geq 2$. Computing a term of $g^{\gamma}=g_{1}^{\gamma_{1}} g_{2}^{\gamma_{2}} \cdots g_{j-1}^{\gamma_{j-1}}$ involves making a choice of $\gamma_{1}$ terms from $g_{1}$ (necessarily each of these will be $b_{1} z_{1}$ ), $\gamma_{2}$ terms from $g_{2}, \gamma_{3}$ terms from $g_{3}$, etc. Since $\lambda_{j}=\lambda_{1}^{\gamma_{1}} \lambda_{2}^{\gamma_{2}} \cdots \lambda_{j-1}^{\gamma_{j-1}}$, making these successive choices produces a j-permissible monomial.

As an example, again suppose as above that $\varphi$ has resonances $\lambda_{2}=\lambda_{1}^{2}$, $\lambda_{3}=\lambda_{1}^{3}=\lambda_{1} \lambda_{2}$ and $\lambda_{4}$ has the resonance relations in Equation (4). In the expansion of $\hat{g}_{4} \circ g$ we obtain, for example, the terms from $g_{2}^{2} g_{3}$ since $(0,2,1,0) \in \Gamma_{4}$. The monomials obtained by choosing two terms from $g_{2}$ (either $b_{2} z_{2}$ or a multiple of $z_{1}^{2}$ ) and one from $g_{3}$ (either $b_{3} z_{3}$, a multiple of $z_{1}^{3}$ or a multiple of $\left.z_{1} z_{2}\right)$ are all in $\Gamma_{4}$.

Since Theorem 3 applies when $\varphi$ has no resonances, in the next two results we consider the resonant case.

Theorem 6. Let $\varphi$ be an analytic self-map of $B_{N}$ fixing 0 and not unitary on any slice with $\varphi^{\prime}(0)$ upper triangular with distinct diagonal entries $\lambda_{j}$ satisfying

$$
1>\left|\lambda_{1}\right| \geq\left|\lambda_{2}\right| \geq \cdots \geq\left|\lambda_{N}\right|>0 .
$$

Suppose $\varphi$ has at least one resonance relation. Let $g \in \mathcal{G}_{\varphi}$ with

$$
g^{\prime}(0)=\operatorname{diag}\left\{b_{1}, b_{2}, \ldots, b_{N}\right\}
$$

and assume that whenever a resonance relation

$$
\lambda_{j}=\lambda_{1}^{k_{1}} \cdots \lambda_{j-1}^{k_{j-1}}
$$

holds then

$$
b_{j} \neq b_{1}^{k_{1}} \cdots b_{j-1}^{k_{j-1}} .
$$

Then there exists $\hat{g} \in \mathcal{G}_{\varphi}$ with $\hat{g}^{\prime}(0)=I$ and $\hat{g} \circ g=g^{\prime}(0) \hat{g}$.

Proof. By hypotheses the coordinate functions of $g$ are

$$
g_{j}\left(z_{1}, \ldots, z_{N}\right)=b_{j} z_{j}+\sum_{\gamma \in \Gamma_{j}} c^{j}(\gamma) z^{\gamma} .
$$


Set

$$
\hat{g}_{j}\left(z_{1}, \ldots, z_{N}\right)=z_{j}+\sum_{\gamma \in \Gamma_{j}} \hat{c}^{j}(\gamma) z^{\gamma}
$$

so that $\hat{g}^{\prime}(0)=I$. We need only show that the coefficients $\hat{c}^{j}(\gamma)$ may be determined so that

$$
\hat{g} \circ g=g^{\prime}(0) \hat{g}
$$

holds. We will determine these coefficients in the order of the multi-indices in $\Gamma_{j}$. For $\gamma=\left(\gamma_{1}, \ldots, \gamma_{j-1}, 0, \ldots, 0\right) \in \Gamma_{j}$, write $b^{\gamma}$ for $b_{1}^{\gamma_{1}} b_{2}^{\gamma_{2}} \cdots b_{j-1}^{\gamma_{j-1}}$.

If $\Gamma_{j}=\emptyset$, then $\hat{g}_{j} \circ g=b_{j} \hat{g}_{j}$ holds automatically. If $\Gamma_{j} \neq \emptyset$, let $\tau_{1}$ be the first multi-index in $\Gamma_{j}$, and compare the coefficients of $z^{\tau_{1}}$ on both sides of

$$
\hat{g}_{j} \circ g=b_{j} \hat{g}_{j}
$$

to obtain

$$
c^{j}\left(\tau_{1}\right)+\hat{c}^{j}\left(\tau_{1}\right) b^{\tau_{1}}=b_{j} \hat{c}^{j}\left(\tau_{1}\right)
$$

which can be solved for the unknown $\hat{c}^{j}\left(\tau_{1}\right)$ since $b_{j} \neq b^{\tau_{1}}$.

Next compare the coefficients of $z^{\tau_{2}}$ in Equation (6), where $\tau_{2}$ is the second multi-index of $\Gamma_{j}$. Only for $\gamma=\tau_{1}, \tau_{2}$ can $g^{\gamma}$ contribute a $z^{\tau_{2}}$ term. Thus we are led to the equation

$$
c^{j}\left(\tau_{2}\right)+b^{\tau_{2}} \hat{c}^{j}\left(\tau_{2}\right)+\cdots=b_{j} \hat{c}^{j}\left(\tau_{2}\right)
$$

where $\cdots$ indicates terms depending only on coefficients of $g$ and/or the just determined value $\hat{c}^{j}\left(\tau_{1}\right)$. This can be solved for $\hat{c}^{j}\left(\tau_{2}\right)$ since $b^{\tau_{2}} \neq b_{j}$. Proceeding in this way through the multi-indices of $\Gamma_{j}$ in the prescribed order we obtain equations

$$
c^{j}\left(\tau_{k}\right)+b^{\tau_{k}} \hat{c}^{j}\left(\tau_{k}\right)+\cdots=b_{j} \hat{c}^{j}\left(\tau_{k}\right)
$$

where the omitted terms on the left are known quantites, possibly involving the coefficients $\hat{c}^{j}\left(\tau_{i}\right)$ where $\tau_{i}$ preceedes $\tau_{k}$.

At this point we have determined $\hat{c}^{j}(\gamma), \gamma \in \Gamma_{j}$ so that in Equation (6) the coefficients of any $z^{\tau}, \tau \in \Gamma_{j}$ agree on both sides. Recall that by Lemma 5 the monomials of order at least two which appear in the expansion of the left side of Equation (6) are all j-permissible, so in fact we have shown that the coefficients of $z^{\tau}$ for any multi-index $\tau$ of total order at least two on both sides of the equation agree. The only non-zero first order terms on either side of Equation (6) are $b_{j} z_{j}$. Hence with the determined values of $\hat{c}^{j}(\gamma)$, Equation (5) holds.

Theorem 7. Let $\varphi: B_{N} \rightarrow B_{N}$ be analytic such that $\varphi(0)=0, \varphi$ is not unitary on any slice, and $A=\varphi^{\prime}(0)$ is upper triangular with distinct diagonal entries $\lambda_{j}$ satisfying $1>\left|\lambda_{1}\right| \geq\left|\lambda_{2}\right| \geq \cdots \geq\left|\lambda_{N}\right|>0$. Assume that $\varphi$ has resonances so $\Gamma_{j}$ is non-empty for at least one $j$. Suppose $\varphi$ has a Schroeder map $\sigma_{\varphi}$ with $\sigma_{\varphi}^{\prime}(0)=I$. If $\varphi \circ \psi=\psi \circ \varphi$ for some analytic self-map $\psi$ of 
$B_{N}$, and the resonances of $\varphi$ are not also resonances of $\psi$, then $\varphi$ and $\psi$ have a common Schroeder map which is locally univalent near 0.

Before giving the proof, we clarify the meaning of the hypothesis "the resonances of $\varphi$ are not also resonances of $\psi$ ". Since $\varphi$ and $\psi$ commute, so do $\varphi^{\prime}(0)$ and $\psi^{\prime}(0)$. Since $\varphi^{\prime}(0)$ is assumed to have distinct eigenvalues, this means that $\varphi^{\prime}(0)$ and $\psi^{\prime}(0)$ may be simultaneously diagonalized, and we may find an $N \times N$ invertible matrix $S$ so that

$$
S \varphi^{\prime}(0) S^{-1}=\operatorname{diag}\left(\lambda_{1}, \lambda_{2}, \ldots, \lambda_{N}\right)
$$

and

$$
S \psi^{\prime}(0) S^{-1}=\operatorname{diag}\left(\mu_{1}, \mu_{2}, \ldots, \mu_{N}\right)
$$

where the $\lambda_{j}$ 's appear in non-increasing order, but there is no apriori ordering on the $\mu_{j}$ 's. To say that the resonances of $\varphi$ are not also resonances of $\psi$ means that if

$$
\lambda_{j}=\lambda_{1}^{k_{1}} \cdots \lambda_{j-1}^{k_{j-1}}
$$

then

$$
\mu_{j} \neq \mu_{1}^{k_{1}} \cdots \mu_{j-1}^{k_{j-1}}
$$

(with the given ordering on the $\mu_{j}^{\prime} s$ ).

Proof. If $\varphi \circ \psi=\psi \circ \varphi$ we have already observed that $\sigma_{\varphi} \circ \psi$ is a Schroeder map for $\varphi$. By Corollary 2, we must have

$$
\sigma_{\varphi} \circ \psi=S^{-1} \circ g \circ S \circ \sigma_{\varphi}
$$

where $S$ diagonalizes $\varphi^{\prime}(0)$ and $\psi^{\prime}(0)$ as just described and $g \in \mathcal{G}_{\varphi}$ so that the coordinate functions of $g$ are

$$
g_{j}\left(z_{1}, \ldots, z_{N}\right)=b_{j} z_{j}+\sum_{\gamma \in \Gamma_{j}} c^{j}(\gamma) z^{\gamma} .
$$

Upon differentiation of the relation $\sigma_{\varphi} \circ \psi=S^{-1} g S \sigma_{\varphi}$ we see that $\psi^{\prime}(0)=$ $S^{-1} g^{\prime}(0) S$ so that

$$
\operatorname{diag}\left(\mu_{1}, \ldots, \mu_{N}\right)=S \psi^{\prime}(0) S^{-1}=g^{\prime}(0)=\operatorname{diag}\left(b_{1}, \ldots, b_{N}\right)
$$

and by hypothesis $\lambda_{j}=\lambda_{1}^{k_{1}} \cdots \lambda_{j-1}^{k_{j-1}} \Rightarrow b_{j} \neq b_{1}^{k_{1}} \cdots b_{j-1}^{k_{j-1}}$. By Theorem 6, there exists $\hat{g} \in \mathcal{G}_{\varphi}$ with $\hat{g}^{\prime}(0)=I$ and $\hat{g} \circ g=g^{\prime}(0) \hat{g}$. By Corollary 2, $S^{-1} \hat{g} S \sigma_{\varphi}$ is a Schroeder map for $\varphi$; its derivative at 0 is $I$. The following calculation shows that it is also a Schroeder map for $\psi$ :

$$
\begin{aligned}
\left(S^{-1} \hat{g} S \sigma_{\varphi}\right) \psi=S^{-1} \hat{g} S S^{-1} g S \sigma_{\varphi} & =S^{-1} \hat{g} g S \sigma_{\varphi} \\
& =S^{-1} g^{\prime}(0) \hat{g} S \sigma_{\varphi} \\
& =\left(S^{-1} g^{\prime}(0) S\right) S^{-1} \hat{g} S \sigma_{\varphi} \\
& =\psi^{\prime}(0)\left(S^{-1} \hat{g} S \sigma_{\varphi}\right) .
\end{aligned}
$$


In Example 1 we saw that Theorem 7 can fail if the resonances of $\varphi$ are also resonances of $\psi$. Of course, if $\psi$ is a natural iterate of $\varphi$, then $\varphi$ and $\psi$ will commute, have the same resonances, and have a common Schroeder map.

One application of Theorems 7 and 3 is to extract qualitative information about the maps which commute with a given map. Our next theorem is a result in this direction. It depends on the following result from [2].

Proposition 8 ([2]). Let $\varphi$ be an analytic map of $B_{N}$ into itself with $\varphi(0)=$ 0 and $A=\varphi^{\prime}(0)$ invertible and suppose $\varphi$ is not unitary on any slice of $B_{N}$. If $\sigma_{\varphi}$ is an analytic map of $B_{N}$ into $\mathbf{C}^{N}$ that solves Schroeder's functional equation $\sigma_{\varphi} \circ \varphi=A f$ and $\sigma_{\varphi}^{\prime}(0)$ is invertible, then $\sigma_{\varphi}$ is univalent on $B_{N}$ if and only if $\varphi$ is univalent on $B_{N}$.

Corollary 9. Suppose $\varphi$ and $\psi$ are commuting analytic self-maps of $B_{N}$, both fixing 0 , not unitary on any slice, and having invertible derivative at 0. Suppose further that they satisfy the hypotheses of either Theorem 3 or Theorem 7. Then if $\varphi$ is univalent in $B_{N}$ so is $\psi$.

Proof. There is a common locally univalent Schroeder map for $\varphi$ and $\psi$ which by the "if" direction of Proposition 8 is univalent in $B_{N}$. Now apply the "only if" direction of the proposition for $\psi$ to conclude that $\psi$ is univalent in $B_{N}$.

The invertibility of $\varphi^{\prime}(0)$ and $\psi^{\prime}(0)$ is necessary in this corollary, as the maps $\varphi\left(z_{1}, z_{2}\right)=\left(1 / 2 z_{1}, 1 / 3 z_{2}\right)$ and $\psi\left(z_{1}, z_{2}\right)=\left(1 / 2 z_{1}, 0\right)$ which share the Schroeder map $\sigma\left(z_{1}, z_{2}\right)=\left(z_{1}, z_{2}\right)$ show.

Finally, we observe that our proof of Theorem 7 depends on the hypothesis that the eigenvalues of $\varphi^{\prime}(0)$ are distinct. This hypothesis plays a significant role in Theorem 6 as it means each coordinate function $g_{j}$ has at most one non-zero linear term. We leave consideration of the repeated eigenvalue case for a later time.

\section{References}

[1] C.C. Cowen, Commuting analytic functions, Trans. Amer. Math. Soc., 283 (1984), 685-695.

[2] C.C. Cowen and B.D. MacCluer, Schroeder's equation in several variables, preprint, 1998.

[3] G. Koenigs, Recherches sur les integrales de certaines equations fonctionnelles, Ann. Sci. Ecole Norm. Sup., Ser. 3, 1 (1884), 3-41.

[4] W. Pranger, Iteration of functions analytic on a disk, Aequationes Math., 4(1) (1970), 201-204. 
[5] W. Rudin, Function Theory in the Unit Ball of $C^{n}$, Grundlehren der math. Wiss., 241, Springer-Verlag, New York, 1980.

Received August 11, 1998 and revised July 6, 1999.

\section{UNIVERSITY OF VIRGINIA}

Charlottesville, VA 22903-4137

E-mail address: maccluer@virginia.edu 